\title{
Multi-dimensional Legendre wavelet matrix approach for hyperbolic telegraph equation with dirichlet boundary condition
}

\author{
Vijay Patel ${ }^{1}$ and Dhirendra Bahuguna ${ }^{1}$ \\ ${ }^{1}$ Indian Institute of Technology Kanpur
}

May 5, 2020

\begin{abstract}
The present article is devoted to developing the Legendre wavelet operational matrix method (LWOMM) to find the numerical solution of two-dimensional hyperbolic telegraph equations (HTE) with appropriate initial time boundary space conditions. The Legendre wavelets series with unknown coefficients has used for approximating the solution in both of the spatial and temporal variables. The basic idea for discretizing two-dimensional HTE is based on differentiation and integration of operational matrices. By implementing LWOMM on HTE, HTE is transformed into algebraic generalized Sylvester equation. Numerical experiments are provided to illustrate the accuracy and efficiency of the presented numerical scheme. Comparisons of numerical results associated with the proposed method with some of the existing numerical methods confirm that the method is easy, accurate and fast experimentally. Moreover, we have investigated the convergence analysis of multidimensional Legendre wavelet approximation.
\end{abstract}

\section{Hosted file}

MMAS_Vijay_20_02_2020.pdf available at https://authorea.com/users/299657/articles/429144multi-dimensional-legendre-wavelet-matrix-approach-for-hyperbolic-telegraph-equationwith-dirichlet-boundary-condition 
Figure 1.1

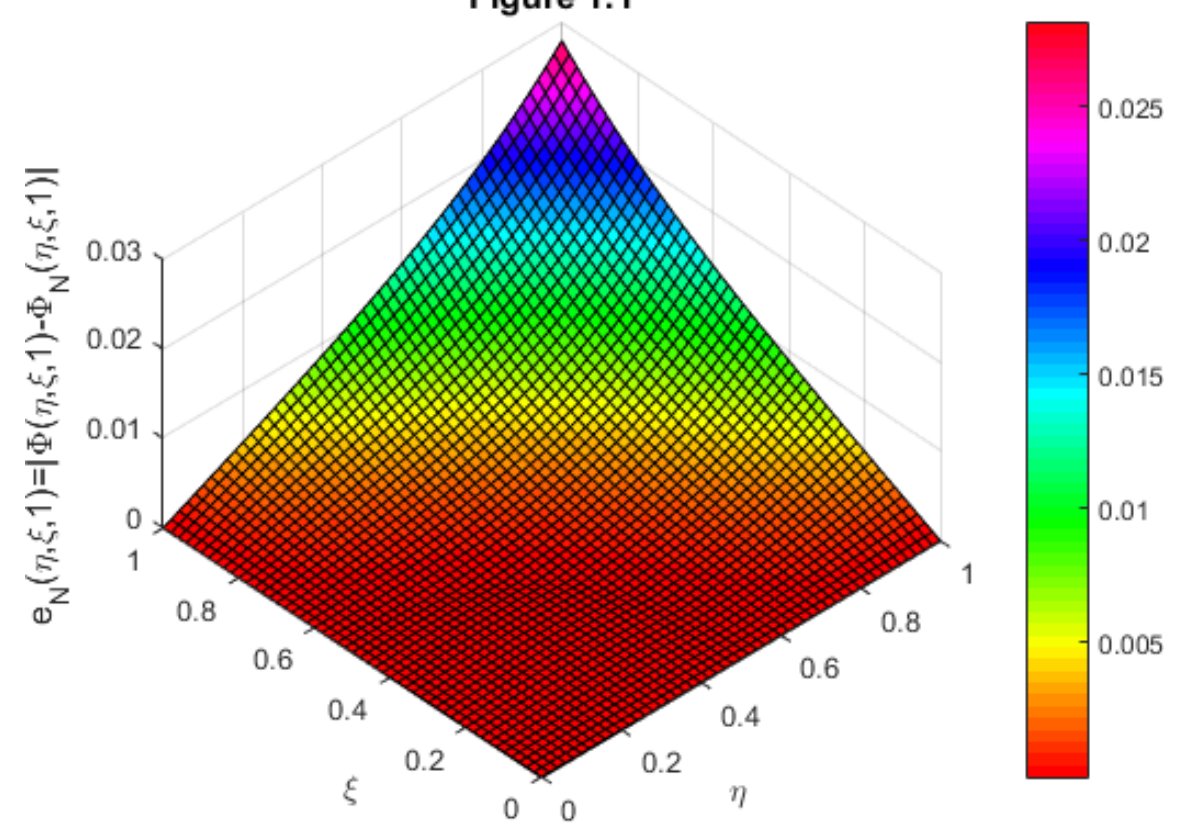

Figure 1.2

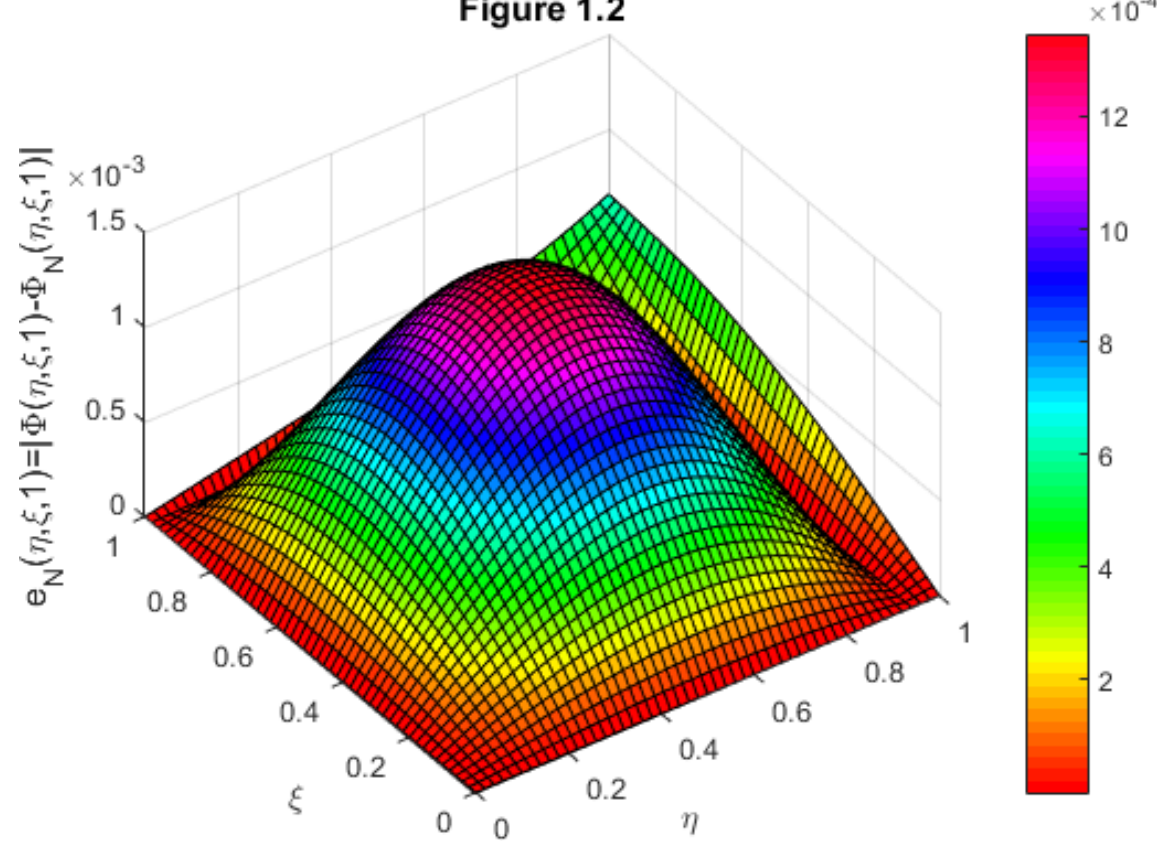


Figure 1.3

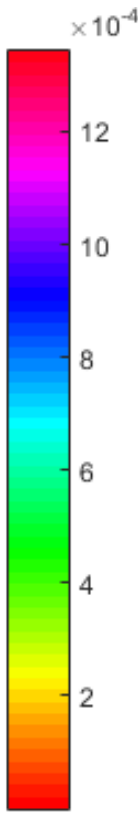

Figure 1.4

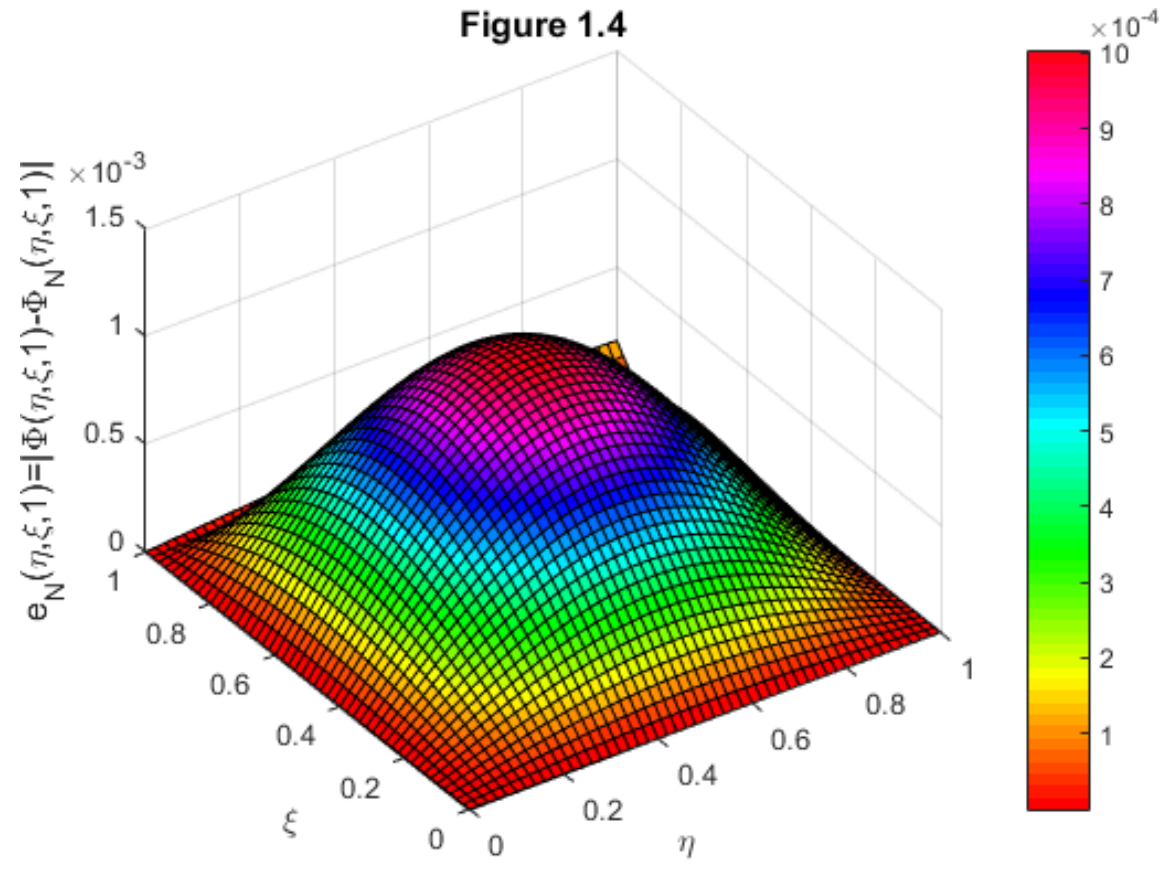


Figure 2.1

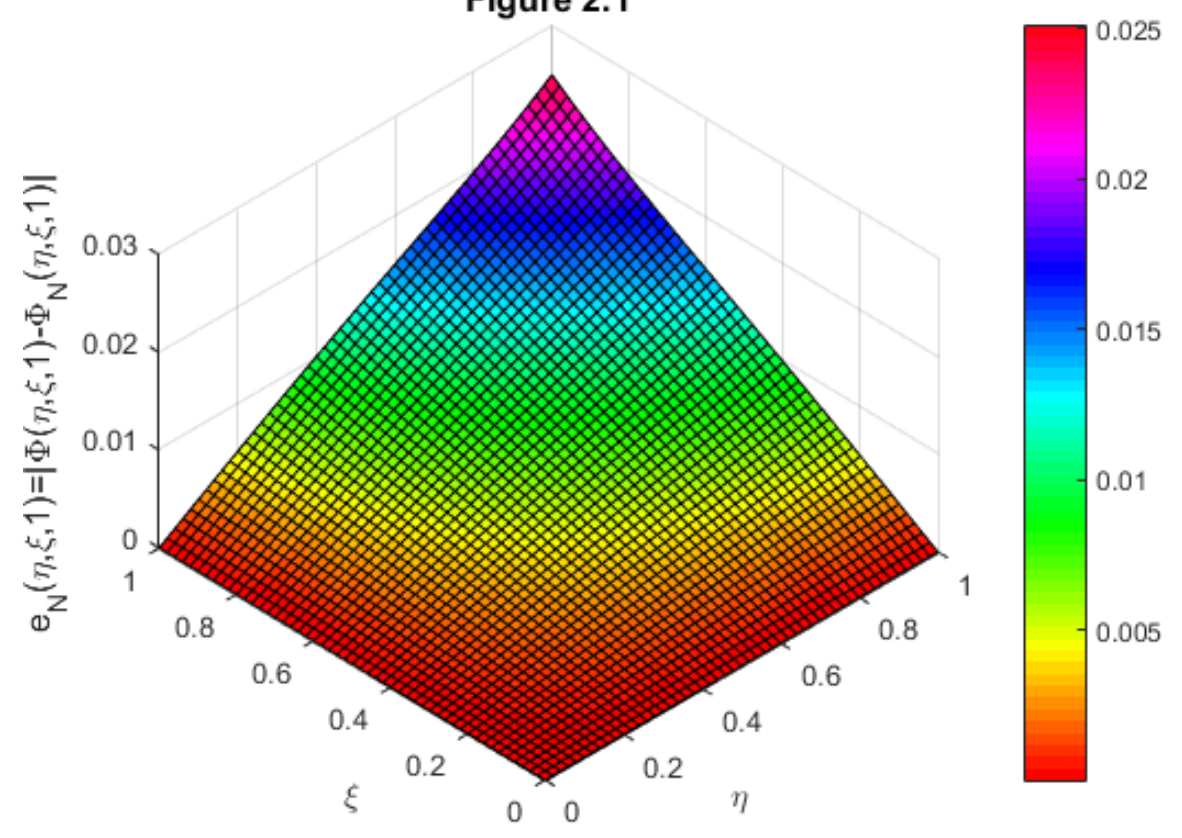

Figure 2.2

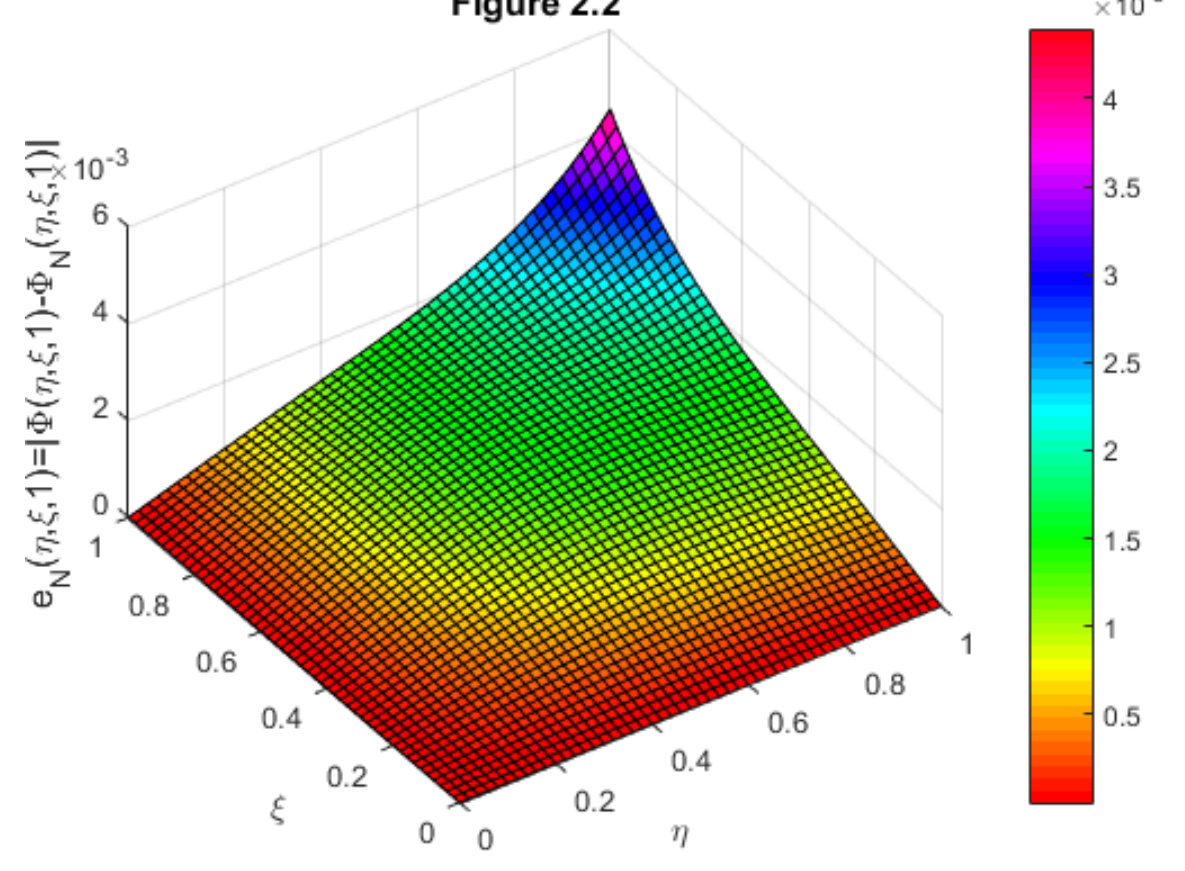


Figure 2.3

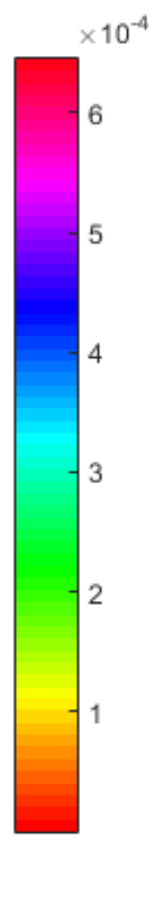

Figure 2.4

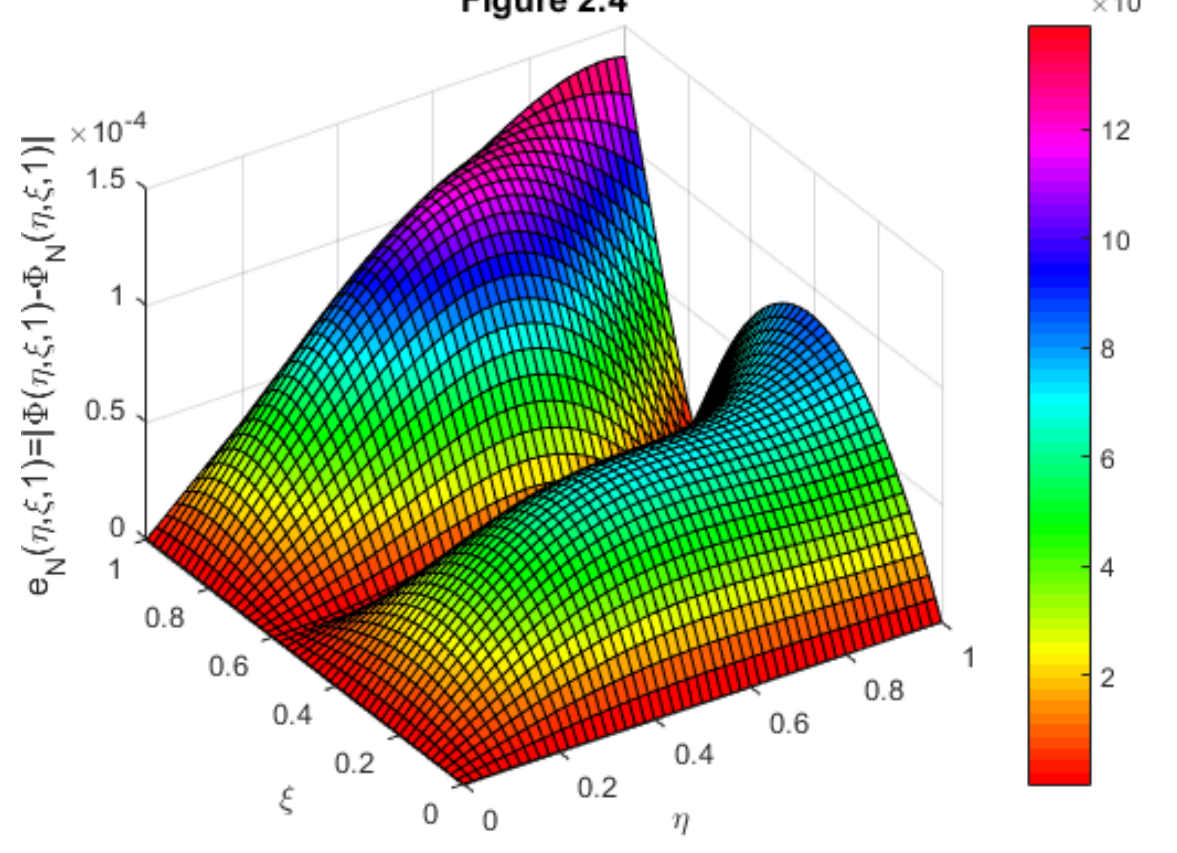


Figure 3.1

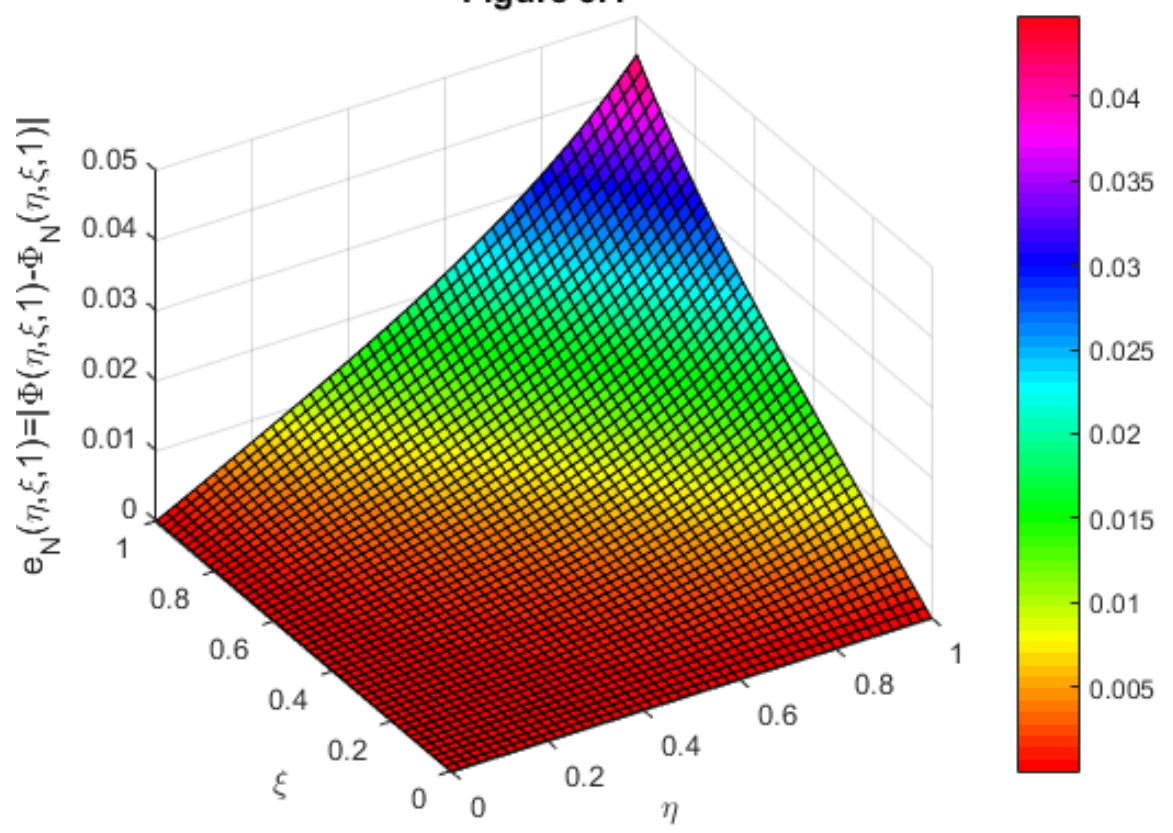

Figure 3.2

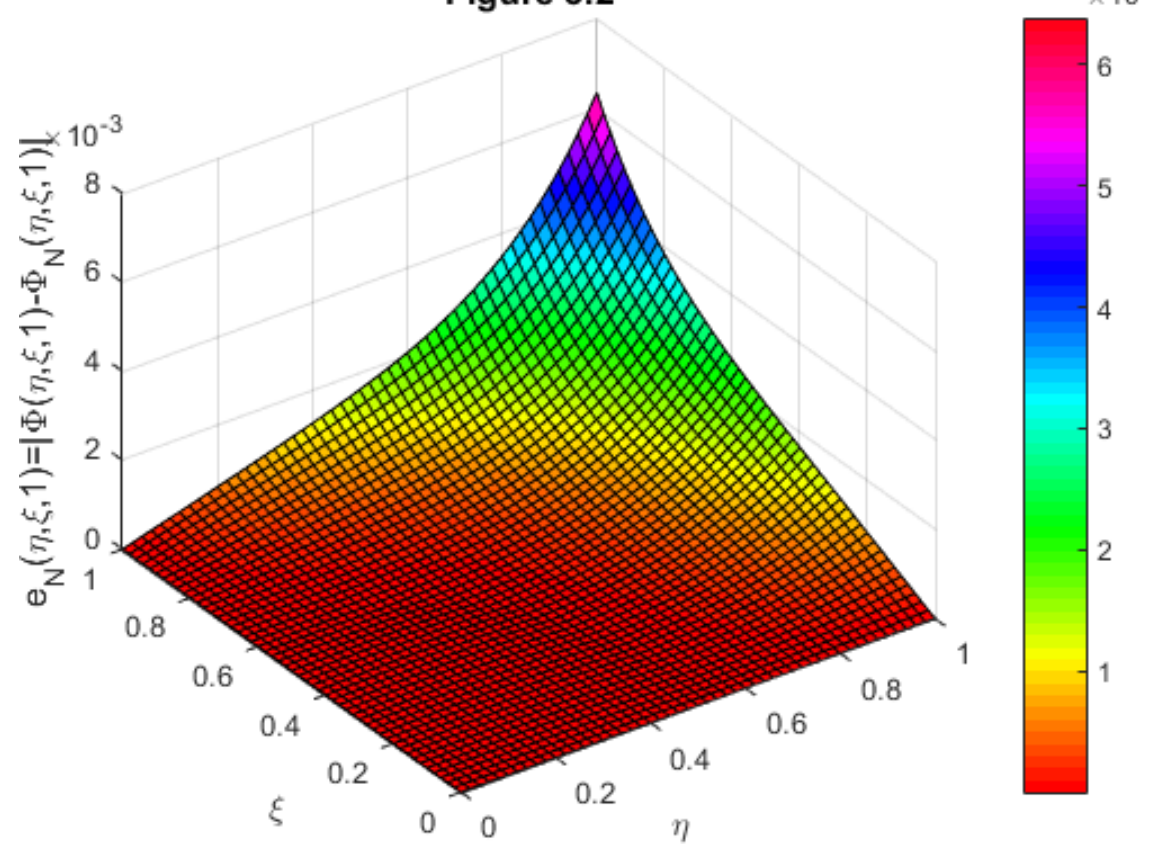


Figure 3.3

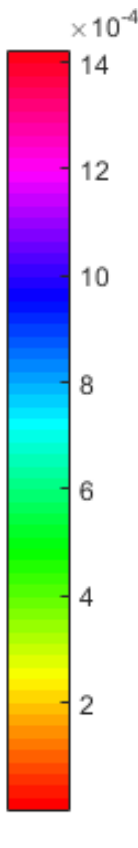

Figure 3.4

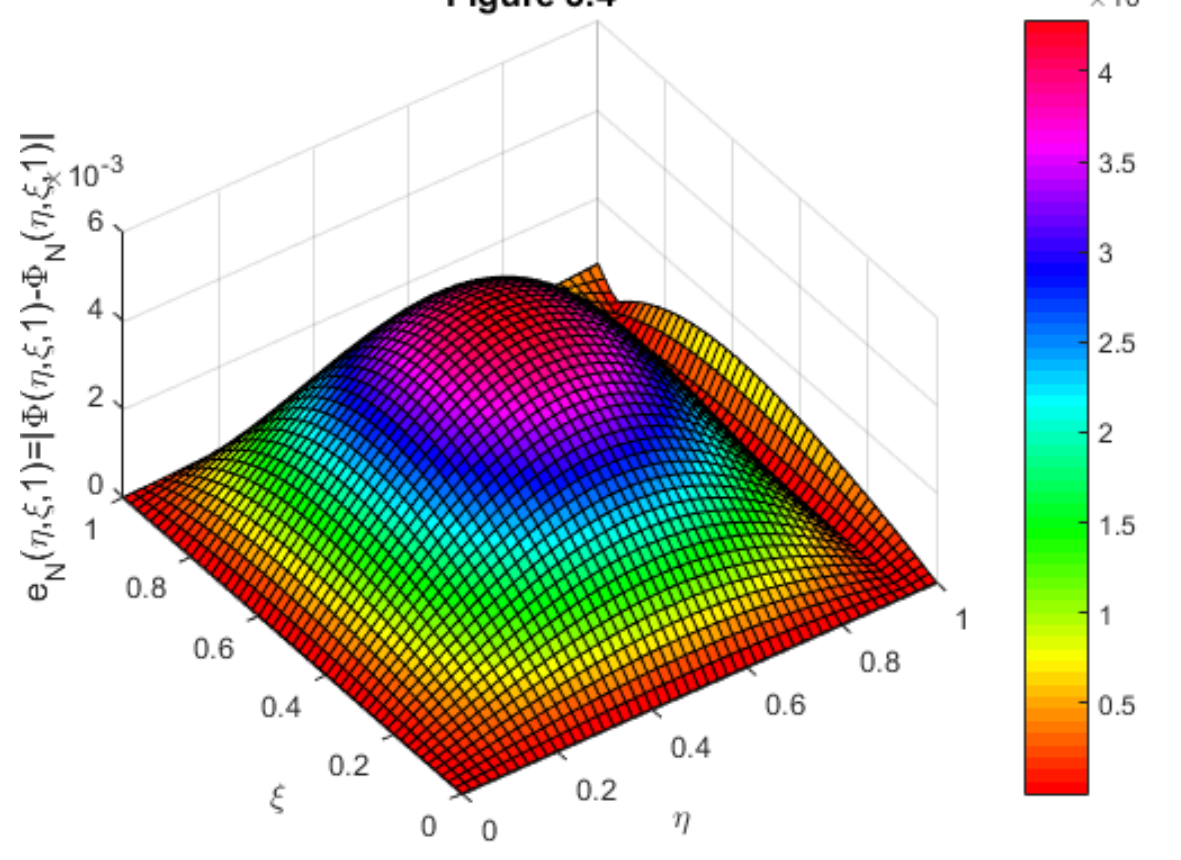

